\title{
Improved experimental resolution of the Vishniac overstability in scaled late-stage supernova remnants
}

\author{
N.J. Riley ${ }^{1}$, S.M. Lewis ${ }^{1}$, M.L. Wisher ${ }^{2}$, M. W. Kimmel ${ }^{2}$, K.W. Struve ${ }^{2}$, J.L. Porter ${ }^{2}$, R.D. Bengtson ${ }^{1}$, T. \\ Ditmire $^{1}$ \\ 1. Center for High Energy Density Science, University of Texas at Austin, Austin, TX, 78712 \\ 2. Sandia National Laboratories, Albuquerque, NM, 87185 \\ Corresponding author: Nathan Riley (njr454@ph.utexas.edu)
}

\section{Abstract}

Radiative shocks and blast waves are important in many astrophysical contexts, such as supernova remnant formation, cosmic ray production, and gamma ray bursts. Structure formation on radiative blast wave fronts in late-stage supernova remnants is expected to play a role in star formation via seeding of the Jeans instability. The origin of these structures is believed to be an instability described theoretically by Vishniac (ApJ 274:152-167, 1983)[1], which has been subject to continued numerical and experimental study. We report here on a series of experiments designed to examine the effect of magnetic fields on the Vishniac overstability. Preliminary results suggest that a strong transverse magnetic field appears to shift the overstability to longer wavelengths, which may have implications for gravitational star formation models. We present unmagnetized results from an experiment in progress which decomposes the spatial structure of the blast wave for quantitative analysis of magnetic and radiative effects.

\section{Keywords}

Hydrodynamics, blast waves, Vishniac overstability, magnetic fields

\section{Introduction}

Shocks are ubiquitous in astrophysical gas dynamics, forming the mechanism for such phenomena as the dynamics of supernova remnant formation, cosmic ray production, and gamma ray bursts. Dynamic instabilities formed in radiative shock fronts are of particular interest, as they are thought to play a role in star formation via seeding of the Jeans instability [1]. Magnetohydrodynamic scaling relations enable the study of these phenomena in the laboratory $[5,6]$, and a significant body of work exists in this area, although until recently such experiments have been purely hydrodynamic [2-4] . We have conducted three runs in an experiment which extends scaled hydrodynamic simulations to incorporate an astrophysically relevant magnetic field. Our preliminary findings using the Texas Petawatt Laser suggest significant effects on both large-scale dynamics and shock stability at astrophysically-relevant field levels. In order to refine these findings, we have undertaken the construction of a target area at the ZBeamlet laser at Sandia National laboratories dedicated to magnetized target experiments. 
We are primarily concerned with supernova remnants in the radiative phase $[7,8]$. This phase is of particular importance in supernova remnants which interact with a dense circumstellar medium, such as type Ib/lc or type IIn supernovae. The circumstellar medium decelerates the blast wave rapidly, converting kinetic energy into radiation. Radiation transport in the postshock gas fundamentally changes the shock structure, which varies depending on the optical mean free path of the gas $[9,10]$. In the limiting case of short optical mean free path compared to the characteristic dimensions of the shock, the radiation transport may be treated in a diffusion approximation. In extreme cases, the shock transition loses its discontinuous character and transitions to a thermal wave. When the optical mean free path is of order of the scale size of the system, a radiative precursor is generated by absorption in the cold upstream gas. This precursor may ionize the gas, and if strong enough, may even generate a secondary shock [11]. Conversely, when the optical mean free path is large compared to characteristic spatial dimensions, the effect of the radiation may be approximated as a constant thermodynamic energy loss in the radiative region, without invoking higher-order transport effects. The latter case is typical of late-stage supernova remnants, and is of primary interest in this work.

In hydrodynamic systems supported by thermal pressure, strong radiative cooling can cause collapse of the pressurized system to high density. This phenomenon is general and is observed in both laboratory and astrophysical systems. Laboratory systems typically radiate via different mechanisms than their astrophysical counterparts, but show similar resulting evolution. In point explosions, which are of interest to this work, the result of radiative collapse is a thin pressure-driven shell expanding into the ambient medium. Pressure-driven thin shells have been shown on both theoretical and experimental bases to be subject to hydrodynamic and thermal instabilities $[8,12,13]$. Prominent among these are the family of thin shell instabilities typified by the linear Vishniac overstability, which is a pressure-driven overstability arising from a misalignment of ram and thermal pressure gradients at the shock front [1]. In both the laboratory and astrophysical cases, radiative processes originating in atomic transitions result in energy transport which cause hydrodynamic structures to become unstable $[14,15]$. The density perturbations resulting from the dynamic instability of the shock front are subject to gravitational instability on longer timescales, which affects the structure of the interstellar medium.

There is evidence that the interstellar magnetic field affects the global structure of supernova remnants. Some of the more dramatic examples are the class of bilaterally-symmetric supernova remnants, whose structure has been interpreted as having a magnetic origin [16]. While there are other mechanisms which can result in the observed symmetry, radio polarimetry measurements appear to support this hypothesis. Synchrotron emission maps, and the distribution of orientation of bilaterally-symmetric remnants with respect to the galactic plane also suggest a magnetic origin.

There is also reason to suspect on theoretical grounds that magnetic fields may influence the stability of the shock front. Although a complete analysis has not been done, Vishniac suggests that magnetic pressure will reduce the range of wavelengths and growth rate of the pressure-driven overstability [1]. Blondin and Wright further point out that efficient field amplification in the postshock region may affect thin-shell instabilities even where the preshock field is dynamically unimportant [7]. Tóth \& Draine have shown stabilizing effects of a transverse magnetic field on a radial instability [17], although their analysis does not apply directly to the case discussed here. Finally, Heitsch et al. [18] have shown magnetic inhibition of the nonlinear pressure driven thin shell instability, although the instability is not completely suppressed. There seems to be general agreement that the magnetic field will have a stabilizing effect, although its significance is not well established by theory. 


\section{Experimental methods}

To investigate the global structure and stability of the shock, we undertook three experimental runs at the Texas Petawatt Laser at UT Austin, and at the Z-Beamlet Laser at Sandia National Laboratories. To satisfy the magnetohydrodynamic scaling relations necessary to simulate a supernova remnant, we generate a spherical shock by irradiating a solid target with a laser. The solid target is placed in contact with a gas in which the blast wave propagates. The ablated target mass is much less than that of the accreted gas in the shock, so the solution is well approximated by the Sedov blast wave problem. The gas is selected according to the desired effect of radiation flux on energy transport. Higher-Z gases have a large number of electronic transitions which act as thermodynamic degrees of freedom when the gas is shock heated, corresponding to Bremsstrahlung losses in supernova remnant shocks. Lower- $Z$ gases have fewer active degrees of freedom from radiative effects, serving as a reference for adiabatic blast wave evolution. In this way we can generate both adiabatic and radiatively-cooled blast waves.

To generate a magnetic field of sufficient strength to affect the blast wave dynamics, we used a megaamp pulsed power system to drive current in a single-turn coil surrounding the gas cell, producing a field which was approximately uniform in time and space on the scale of the evolving blast wave. The pulsed power driver was originally developed at Sandia National Laboratories, and adapted to the Texas Petawatt beamline $[19,20]$. It was later moved to Z-Beamlet and the power feed was redesigned for a dedicated target chamber for laser/pulsed power experiments.

To diagnose the blast wave evolution, time-resolved optical diagnostics were used throughout. At UT, a commercial Nd:YAG laser provided a single probe pulse per shot, which was directed into a dark-field Schlieren telescope. We later upgraded the diagnostic chain to use a digital fast framing camera and picket-fence probe capability developed at Sandia [21], enabling the acquisition of multiple blast wave images per shot. The diagnostic chain was upgraded to a larger aperture, and converted to operate as a bright-field Schlieren system. We were thus able to obtain much higher data quality, which greatly facilitated quantitative analysis.

The first run of the experiment was conducted on the Texas Petawatt laser, a $190 \mathrm{~J}, 170 \mathrm{fs}$ laser based on a mixed-glass architecture with a high bandwidth OPCPA front end. The central wavelength on target is $1057 \mathrm{~nm}$. It is capable of peak powers up to 1.1 Petawatts, although for this experiment the laser was operated in a long-pulse configuration by bypassing the compressor. The pulse length in this configuration was $1.6 \mathrm{~ns}$, with a resulting focused intensity of approximately $10^{15} \mathrm{~W} / \mathrm{cm}^{2}$ for a 100 micron focus. To capitalize on previous engineering work, a pulsed power target chamber designed for a magnetized cluster jet experiment was placed on the TPW long-focus beamline. Although the front end can deliver a $\sim 100 \mathrm{fs}$ probe pulse to either target chamber, the relatively long timescale of the experiment dictated the use of a separately triggered probe laser. A commercial Nd:YAG laser with a measured 4 ns FWHM pulse length was used for this purpose, and probe timing was synchronized electronically through the master trigger system.

The second and third runs were conducted at the Z-Beamlet laser at Sandia National Laboratories [22]. The Z-Beamlet laser can deliver up to $6 \mathrm{~kJ}$ over a $6 \mathrm{~ns}$ pulse at $527 \mathrm{~nm}$. We established a point design scaled to the hydrodynamic parameters of the TPW run which used a $1 \mathrm{~kJ}$ drive energy in a $1 \mathrm{~ns}$ pulse. This resulted in a focused intensity of $10^{14} \mathrm{~W} / \mathrm{cm}^{2}$ with a $1.1 \mathrm{~mm}$ phase plate, which was used to prevent beam filamentation in the target foil. A dedicated target chamber was constructed for pulsed power 
experiments, with a collocated diagnostic laser which could be configured to provide synchronized $1 \mathrm{~ns}$ pulses in a picket fence configuration to illuminate multiframe diagnostics.

The experimental point design requires us to generate a spherical blast wave of high specific energy in a uniform gas of known density, with a specific and constant magnetic field. These conditions, together with the experimental capabilities described above, dictate our target design. To generate the blast wave, we have borrowed a concept from Grun et al. and subsequently modified by Edens, and generate the blast wave by irradiating an overdense solid target in contact with an ambient gas. The requirement of a pulsed-power driven field is difficult to reconcile with the presence of an ambient gas, especially at the density required. The presence of rarefied gas in contact with pulsed power hardware is likely to lead to Paschen breakdown [23]. For this reason, a gas cell is used to isolate the shock ambient from the pulsed power environment.

\subsection{Texas Petawatt}

The first generation of gas cell targets were fielded on the Texas Petawatt laser. The primary design parameter was the target scale. This scale may be determined from the point design and pulsed power performance models, constrained by the performance characteristics of the experimental systems. For the TPW experimental run, covering the range of magnetic fields above and below the $B=1$ threshold with respect to the postshock thermal pressure dictated a characteristic dimension of approximately 1 $\mathrm{cm}$. A coil design was subsequently produced with approximately twice this inner diameter to provide space for the gas cell body and diagnostic beam aperture. A quasi-Helmholtz geometry was selected to allow the field to be as uniform as possible in the experimental volume.

With the scale and rough geometry constrained by the field coil, other design constraints included drive and diagnostic beam access, target placement, and gas containment. These issues are related, as the drive beam is sufficiently intense to preclude passage through transmissive elements. For this reason, the compressor was bypassed, situating the laser-plasma interactions in the long-pulse regime and avoiding ultraintense interactions. In this regime, the laser could be propagated through the gas without significant absorption, self-focusing, or filamentation.

To generate a spherically symmetric blast wave in the gas, three target geometries are possible. In the first case, a hemispherical blast wave may be generated by ablating a foil target from the surface in contact with the gas. This geometry was used by Grun et al. in [2]. It is simple to implement, and particularly to align, but suffers from the drawback that the drive beam must traverse the gas. In the second, the tip of a pin target may be ablated, generating a spherical blast wave in the surrounding gas. This geometry reduces boundary effects from the target foil, although it is also affected by the passage of the drive beam through the gas. Finally, a foil may be ablated from the back surface, driving a shock through it and into the gas. This approach eliminates passage of the laser through the gas. It is complicated by the requirement to completely stop the laser while cleanly driving the shock to the front surface and into the gas.

The first generation gas cells were designed with a pin target in the center of the field coil and diagnostic field of view, and the cell body centered symmetrically around it. To deliver the drive beam to the target pin, we made use of the $\mathrm{f} / 40$ focusing geometry of the Texas Petawatt long-focus beamline. We used a dynamic gas fill to avoid nonlinear effects from traversing an entrance window. As the beam can pass through a narrow aperture of high aspect ratio, which tends to retard gas flow, the cell can contain gas on the scale of a few milliseconds without end windows. To realize this, the evacuated cell was filled 
using a pulsed valve through a large diameter fill pipe $1 \mathrm{~ms}$ before the shot, and the blast wave evolved on a timescale several orders of magnitude shorter than the evacuation time through the ends. For this reason, the background gas may be assumed to be static on the timescale of interaction, and effects such as turbulence may be neglected.

The probe beam was defocused to an intensity that could safely pass through ordinary laser line optics. The probe aperture was made as large as mechanically possible to maximize the resolution of the imaging system. This dimension was constrained by the field coils, limiting the available aperture to $12.7 \mathrm{~mm}$. The diagnostic windows were AR-coated BK7 optical glass, supplied by Optical Filter Source. These were selected due to their $\leq \lambda / 4$ surface flatness tolerance, which is a figure of merit for compatibility with Schlieren imaging.

The field coils were fabricated by pressing a sheet copper blank around a mandrel, and finishing on a CNC mill. The gas cell was constructed entirely of non-conductive materials. Polycarbonate was used for the majority of the components due to its resilience and machinability. Its transparency was also a convenient attribute during rough beam alignment with the chamber open. Nylon fishing line was used for the target pin, providing a solid target upon which to focus the incoming laser.

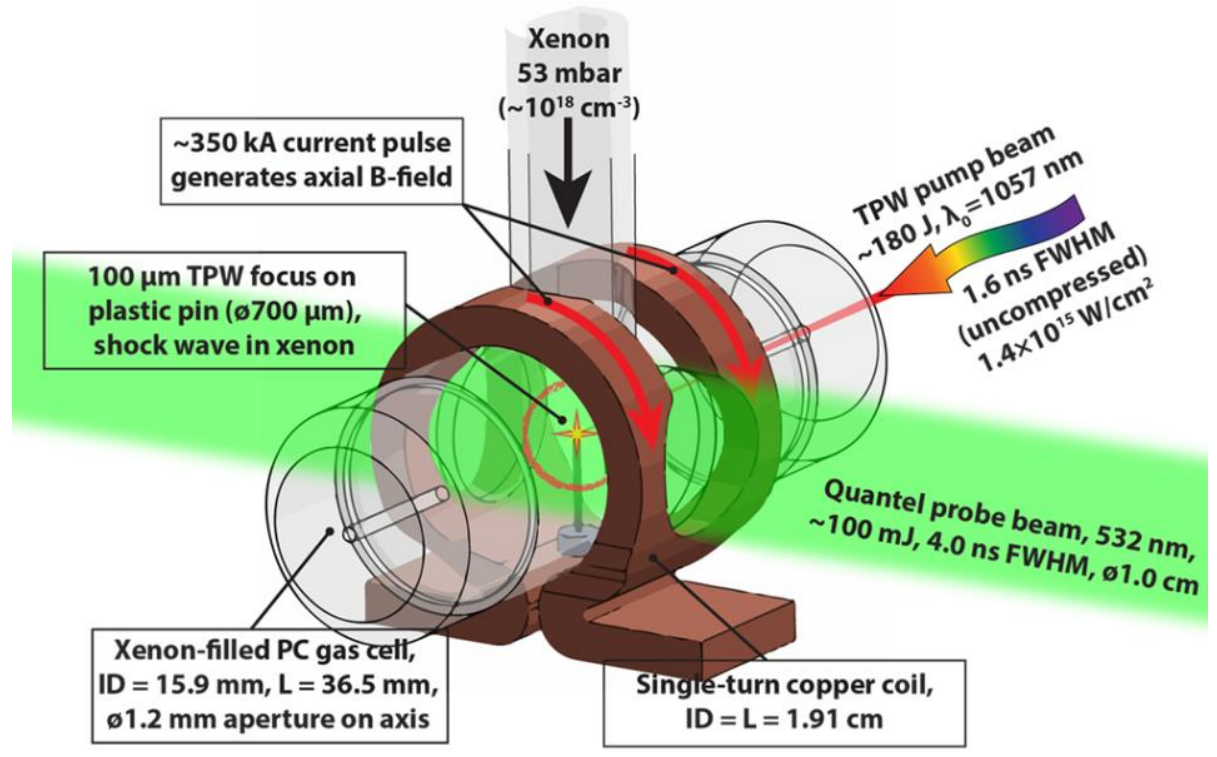

Figure 1. First generation target package as configured for TPW.

\subsection{Z-Beamlet}

The target cell was redesigned to operate on the Z-Beamlet laser due to the $\lesssim f / 10$ focusing geometry of the ZBL target chambers, which was incompatible with the TPW target design. The major change to the target was the choice of geometry. The shorter focal length required by Z-Beamlet meant that placing the focal point inside a pressurized cell was impractical. The remaining option was to deliver the beam to the back surface of a target foil, driving the shock through it and into the gas. With judicious selection 
of the foil material and thickness, this also reduced the interaction of the drive beam with the gas volume, which had complicated the analysis of the Grun and Edens experiments. A static gas fill was used, which simplified the gas handling system at the chamber.

The increased drive energy of Z-Beamlet compared to the Texas Petawatt required a rescaling of the experimental parameters, suggesting a larger scale size for the experiment. This also allowed a larger diagnostic field of view, which yielded greatly improved data quality. The increased radius of the larger field coils limited the maximum magnetic field strength, while still acceptable given previous results obtained at TPW and the expected MagLIF parameter space. Design prototypes of the secondgeneration targets were produced at the original TPW scale, and at double scale. A limited number of prototypes were constructed of both designs, and the double scale configuration was adopted for continued blast wave and preheat experiments.

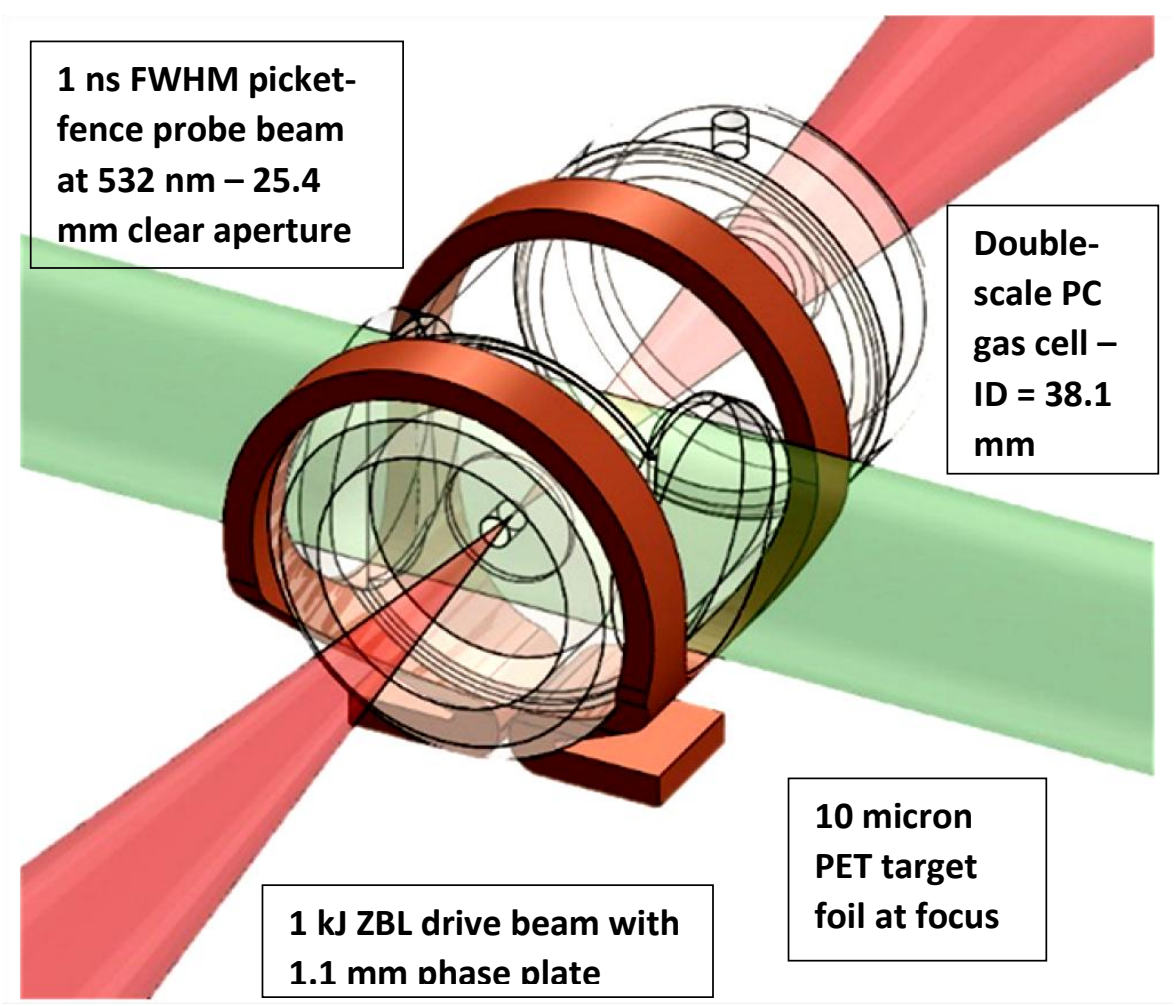

Figure 2. Second generation target package as configured for $Z B L$.

\subsection{Pulsed power}

The pulsed power system used at TPW and ZBL originated in a Sandia LDRD project whose aim was the construction of a small, portable megaamp pulsed power system for use at university facilities, either on its own or as a complement to a larger HEDP facility $[19,20]$. The system utilizes a bank of up to ten capacitor modules, connected in parallel to a magnetically insulated transmission line (MITL). The MITL acts as a vacuum interface, connecting the power hardware to the target chamber. The load bridges the cathode-anode gap of the MITL inside the chamber. In principle, the system can drive any load, such as a Z-pinch, X-pinch, flyer plate, or ramp load. For magnetized laser experiments, a single-turn field coil was used, delivering fields in the range of $\sim 5-20$ T over a period of $\sim 1 \mu \mathrm{s}$. 


\section{Results}

\subsection{Magnetized blast waves}

The Texas Petawatt run was organized around a series of shots at 5,10 , and 15 Tesla nominally. A figure of merit of $B=1$ with respect to the postshock thermal pressure was established for the magnetic field to significantly affect the blast wave dynamics, which corresponded to approximately 5-7 Tesla. This figure relies on a number of assumptions and should be considered to have a large uncertainty, but it was useful to establish a point in design space to enable shots to be taken above and below the threshold.

We present here the shot at each field level which is most clearly interpreted (figure 3). Due to an alignment problem which cut short our available shot time, we had very few usable shots from this run. What we do have should be regarded as extremely preliminary. We therefore present these data as a qualitative guide only, to be confirmed by the continuing ZBL experiments.

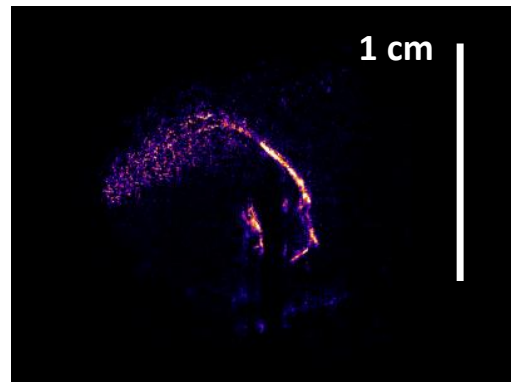

Figure 3a. Blast wave in 40 Torr

Xenon with no magnetic field. Probe

delay is $100 \mathrm{~ns}$. Drive beam enters

from left and strikes target pin in center

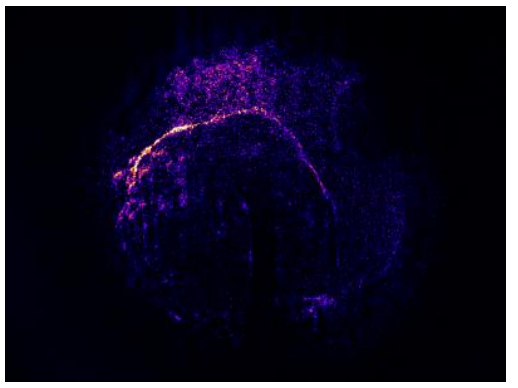

Figure 3b. Blast wave in 40 Torr

Xenon with 5 Tesla magnetic field.

Probe delay is 200 ns. Drive geometry

follows figure $3 a$.

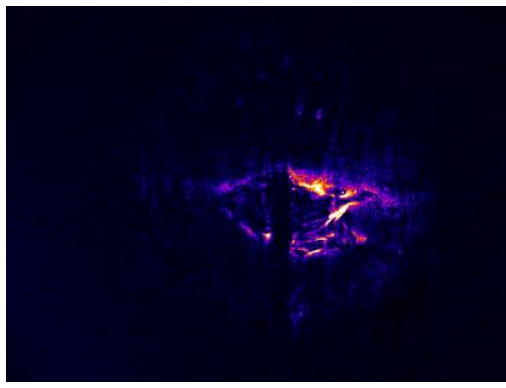

Figure 3c. Blast wave in 40 Torr

Xenon with 14 Tesla magnetic field.

Probe delay is 150 ns. Drive geometry

follows figure $3 a$.

of frame.

The images shown are dark-field Schlieren images. For the reader who may be unfamiliar with the interpretation of Schlieren images, strong density gradients are resolved as bright objects on a dark background[24]. A horizontal knife edge was used, which results in sensitivity to density gradients taken in the vertical direction in the image plane. The practical effect is the appearance of directional illumination of the blast wave from the upper edge of the image plane. The drive beam enters each image from the left, striking the target pin in the center of the image. The blast wave is assumed to be approximately axisymmetric about the beam axis, and the magnetic field, if present, is quasi-uniform and coaligned with the beam axis. For clarification of the experimental orientation, see figure 1.

The blast wave generated without the applied magnetic field, shown in figure 3a, was quasi-spherical, and is highly perturbed at small scales. These features are consistent with previous experimental results $[2,25]$. 
The highest quality shot at 5 Tesla is shown in figure $3 \mathrm{~b}$. It is visibly morphologically distinct from the unmagnetized shot. While the blast wave remains approximately spherically symmetric, the small-scale perturbations appear to be much diminished, and primarily concentrated in the forward quadrant of the blast wave along the beam axis. Notably, this is in the region where the magnetic field is most closely normal to the shock. In the region of the blast wave which is approximately parallel to the magnetic field, the blast wave appears almost smooth, although a long-wavelength perturbation appears to exist.

The highest quality shot at the maximum field of 14 Tesla is shown in figure 3c. The magnetic field at this level is expected to dominate the dynamic pressure in the flow. Blast waves at this field strength have a significantly different morphology than those at lower fields, and are no longer spherically symmetric. There appear to be signs of a filamentary instability along the beam axis.

The shots taken at TPW are suggestive, and appear to show gross magnetic effects corresponding to those expected. The scatter in the probe delay is a serious concern, however, and the data quality precludes quantitative analysis. We attempted to use the blast wave analysis code described below to decompose the projected edge of the blast wave in the spherical harmonic basis, but the images were too noisy to reliably extract it. We therefore designed the next phases of the experiment at ZBL to incorporate a greatly improved diagnostic capability.

\subsection{Quantitative blast wave analysis}

Difficulties in interpreting many of the TPW shots led us to examine alternative approaches to the classical Toepler knife-edge technique, and we experimented with both dark- and bright-field Schlieren methods. We settled on a bright-field method which uses the aperture of the imaging system as the Fourier mask, which is uniquely suitable to the low f-number focusing optics which the geometry of the experiment required. In conjunction with the development of the Schlieren optics, we integrated the UXI multiframe imagers and the Chaco picket-fence probe laser to obtain a series of four to eight blast wave images per shot.

To verify the new target design and diagnostic performance, we undertook a benchmarking run to examine unmagnetized blast waves in neon and xenon in order to derive quantitative information about their spatial structure and stability. This facilitated comparison to the original Grun experiments [2], and allowed us to validate the diagnostics and analysis codes in a context with clear theoretical interpretation and experimental support.

Figure 4 shows a blast wave generated by a $1 \mathrm{~kJ}$ ZBL shot in neon at a chamber pressure of 10 Torr, which at the design scale length is hydrodynamically equivalent to the TPW experiment. The resulting blast wave was elliptical in overall conformation and otherwise nearly unperturbed. This result is in accord with theoretical expectations and previous experimental data. The departure from spherical symmetry may be explained by the $1.1 \mathrm{~mm}$ focal spot size of the laser, which breaks the spherical symmetry by transferring longitudinal momentum through the foil.

The corresponding shot in xenon is shown in figure 5. Experimental conditions are identical to those in figure 4 except for the target gas. The blast wave was highly perturbed at small length scales, which consistent with the Vishniac overstability [2]. For clarity in the analysis to follow, we emphasize that the hydrodynamic parameters of the shots shown below are equivalent to those in the TPW shots under the scaling transformations described in $[5,6]$. To the extent that the hydrodynamics can be considered ideal, the shot shown in figure 5 corresponds to shot 7055 in figure 3 a described earlier. 

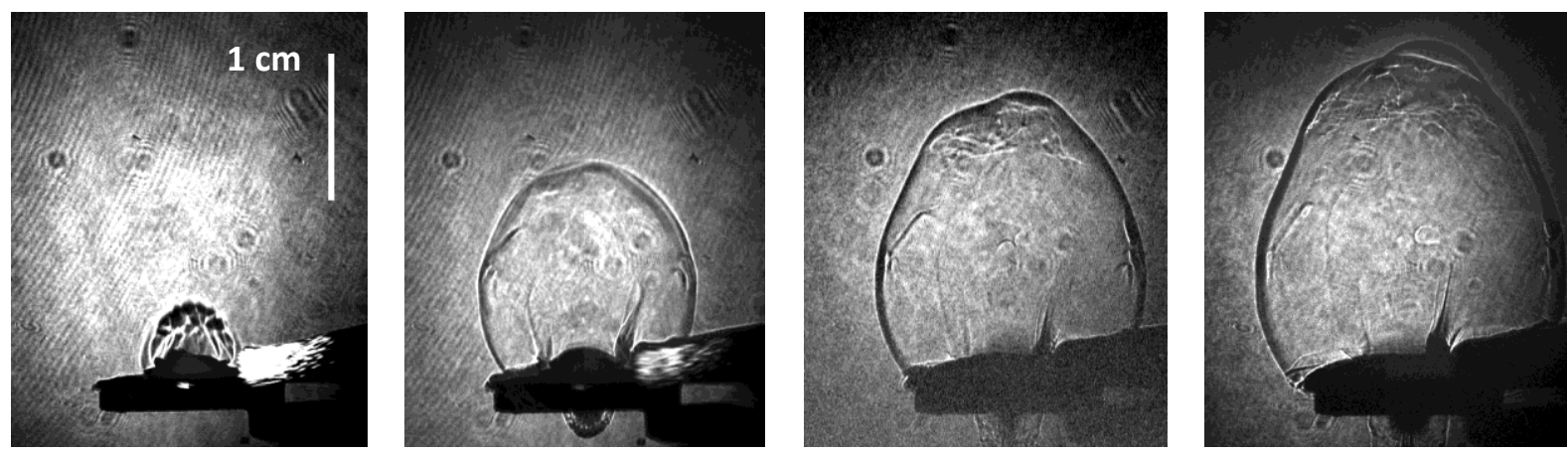

Figure 3. Blast wave in 10 Torr neon. Laser strikes target foil from below. Frame timing is 8345884 ns from drive pulse respectively.
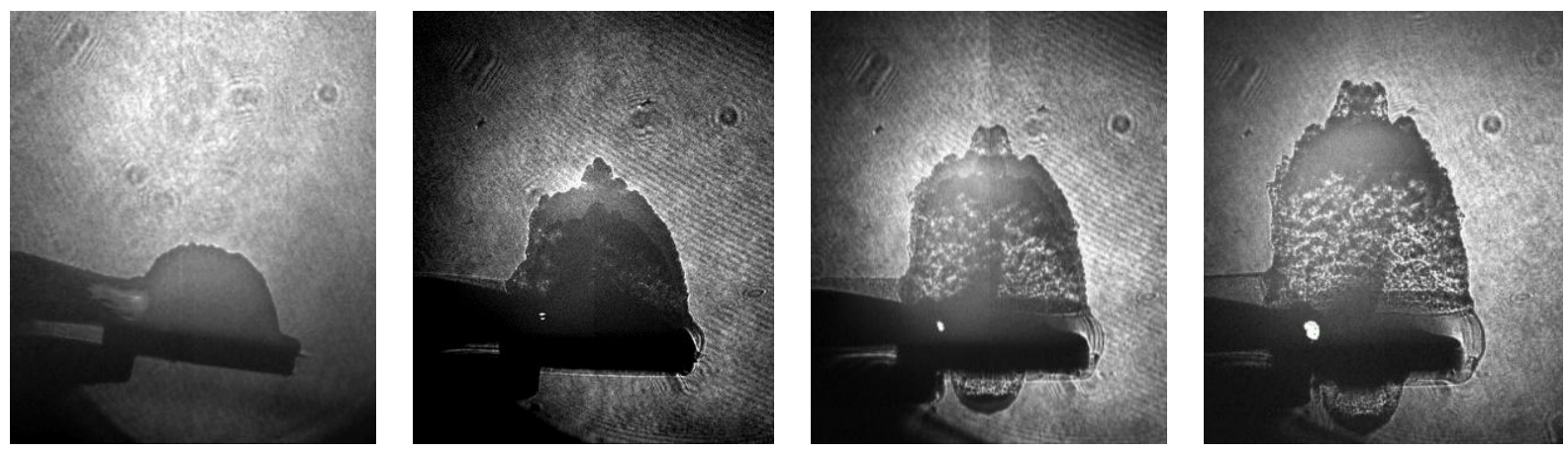

Figure 4. Blast wave in 10 Torr xenon. Laser strikes target foil from below. Frame timing is $3484134184 \mathrm{~ns}$ from

drive pulse respectively.

To quantify the effect of radiation on the global blast wave dynamics, we examined the radial trajectory of the blast wave. In the cases of interest here, there was no uniquely defined radius. In order to get an approximate measure of radial dimension, we averaged the radius of the blast wave over the visible polar angle. The averaged radii of the neon and xenon blast waves were then fitted to a power law and compared. Due to the departure from spherical symmetry, we are less concerned with the precise value of the power-law exponent, but we see that the xenon blast wave shows the expected radiative energy loss (figure 6). 


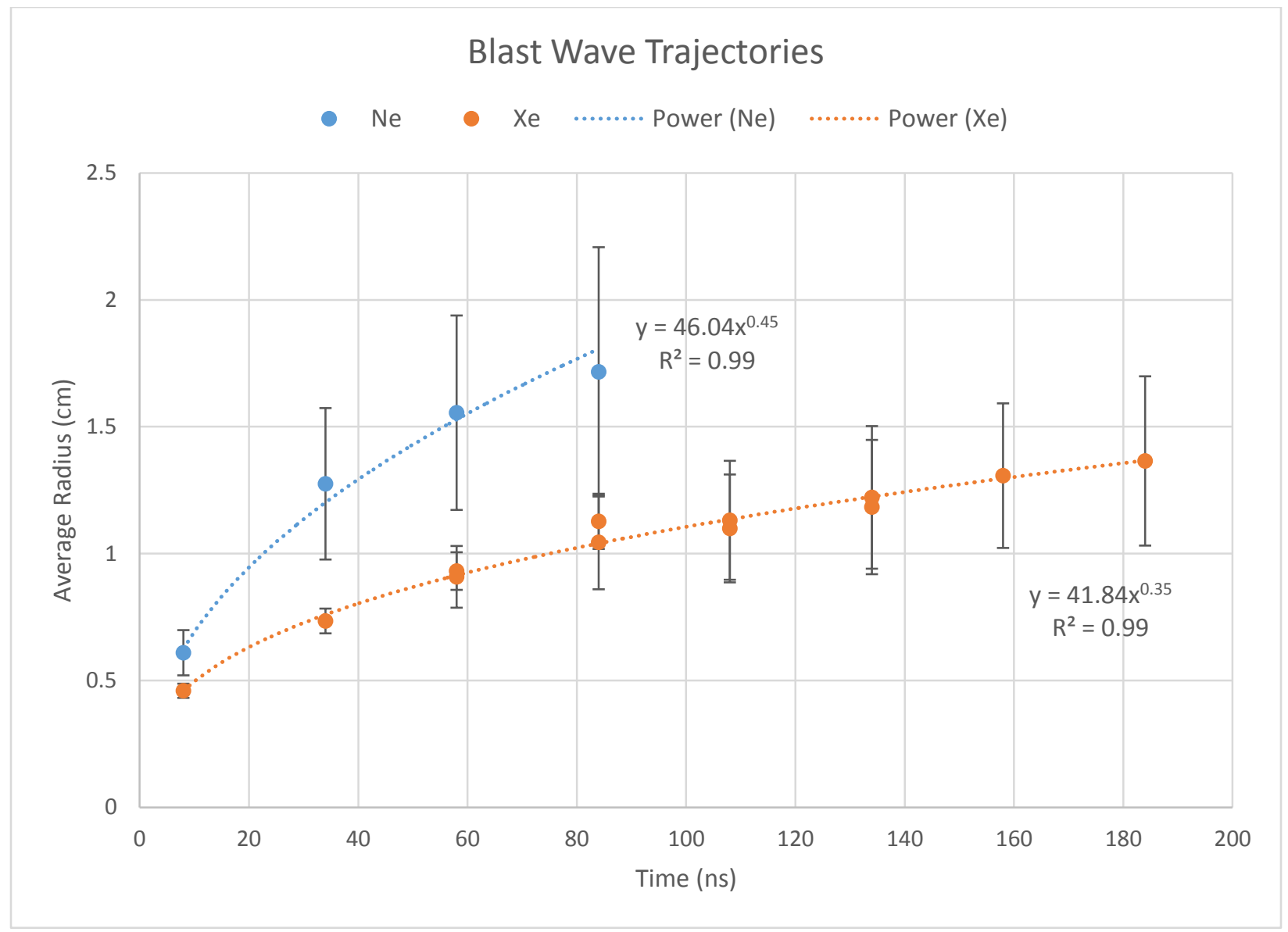

Figure 5. Trajectories of average radius of blast wave in neon and xenon.

We examined the effects of radiative cooling in more detail by decomposing the fine structure of the shock front. To do this, we implemented a software pipeline which extracted the edge of the blast wave from the UXI images, unwrapped it in polar coordinates, subtracted the overall blast wave shape in order to isolate the perturbations, and Fourier transformed the result (figure 7). The Fourier transform was normalized to correspond to the I-component of the spherical harmonic decomposition in Vishniac's theoretical analysis [1]. The output is a spatial mode spectrum for each snapshot in time, from which the dominant spatial modes can be extracted. It was also possible to calculate the growth rate of the spatial modes in some cases, although the noise in the data complicates the analysis. The results are shown in figures 8 and 9, corresponding to the neon and xenon shots shown. The interpretation of the spectra is unavoidably confounded by the elliptical structure of the blast waves, which introduces a continuous spectral background concentrated in low mode numbers and independent of any other structure in the shock front. Nevertheless, we can determine salient features of the mode structure by comparing blast waves under differing experimental conditions. 




Figure 6. Schematic illustration of image analysis pipeline.

Figure 8 shows the spatial mode spectra of the neon shot, where small perturbative structure is present and residuals from the fitting algorithm appear. The result shown can therefore be considered a good reference example of the residual mode spectrum of a nearly unperturbed blast wave. Although the residual spectrum is continuous, as a rule of thumb in later analysis we generally assume that mode content below a mode number of about 10 is an artifact of the large-scale ellipsoidal structure of the blast wave. 


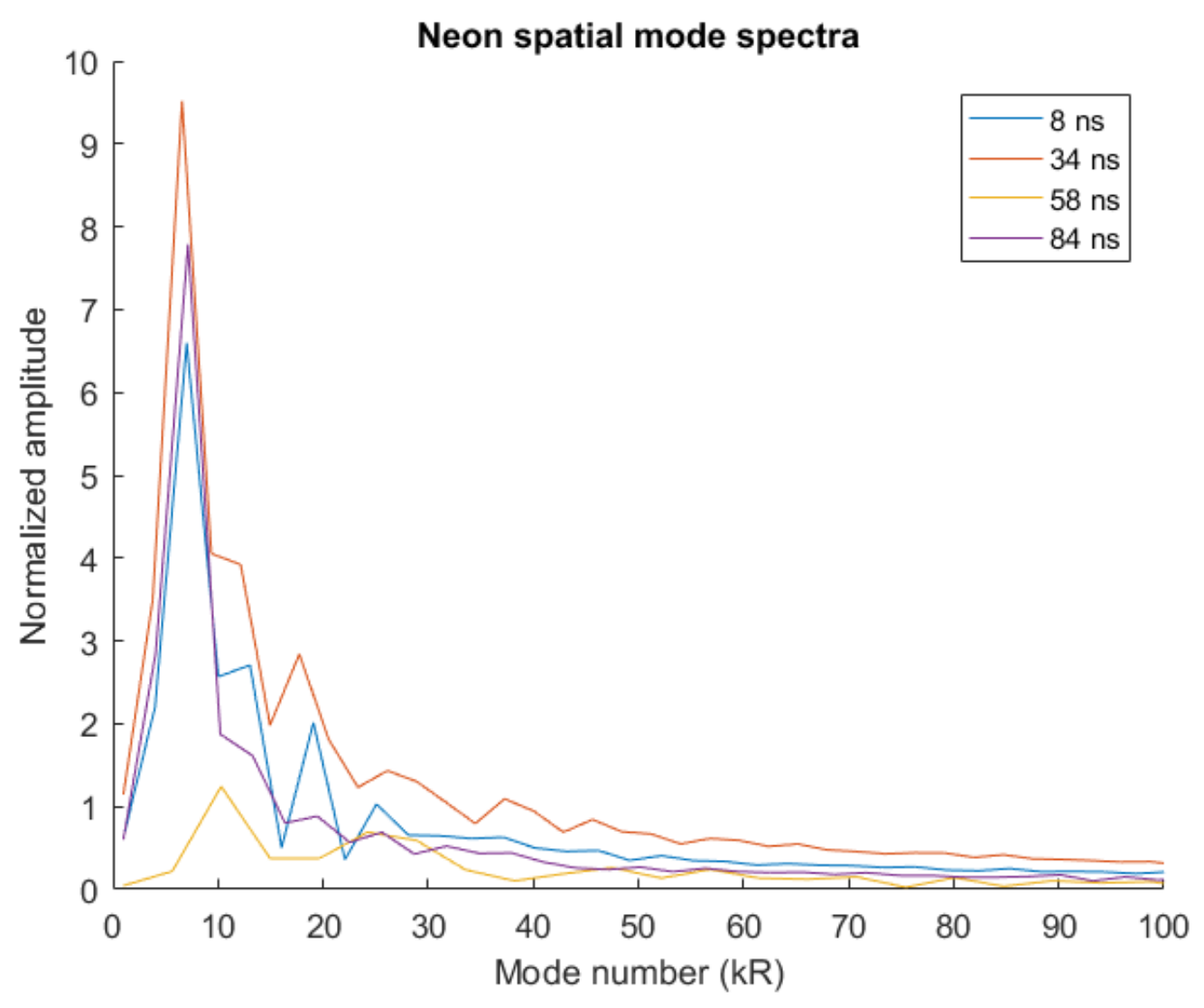

Figure 7. Spatial mode spectra of Neon blast wave.

Figure 9 shows the spatial mode spectra of the xenon shot, taken over eight frames due to the slower time evolution of the shock. As discussed above, we assumed that mode content below mode number $\sim 10$ was background. A peak at mode number 16-20 dominates the spectrum, with a continuous distribution up to a mode number of 60 which appears to be above the background implied by figure 8 . 


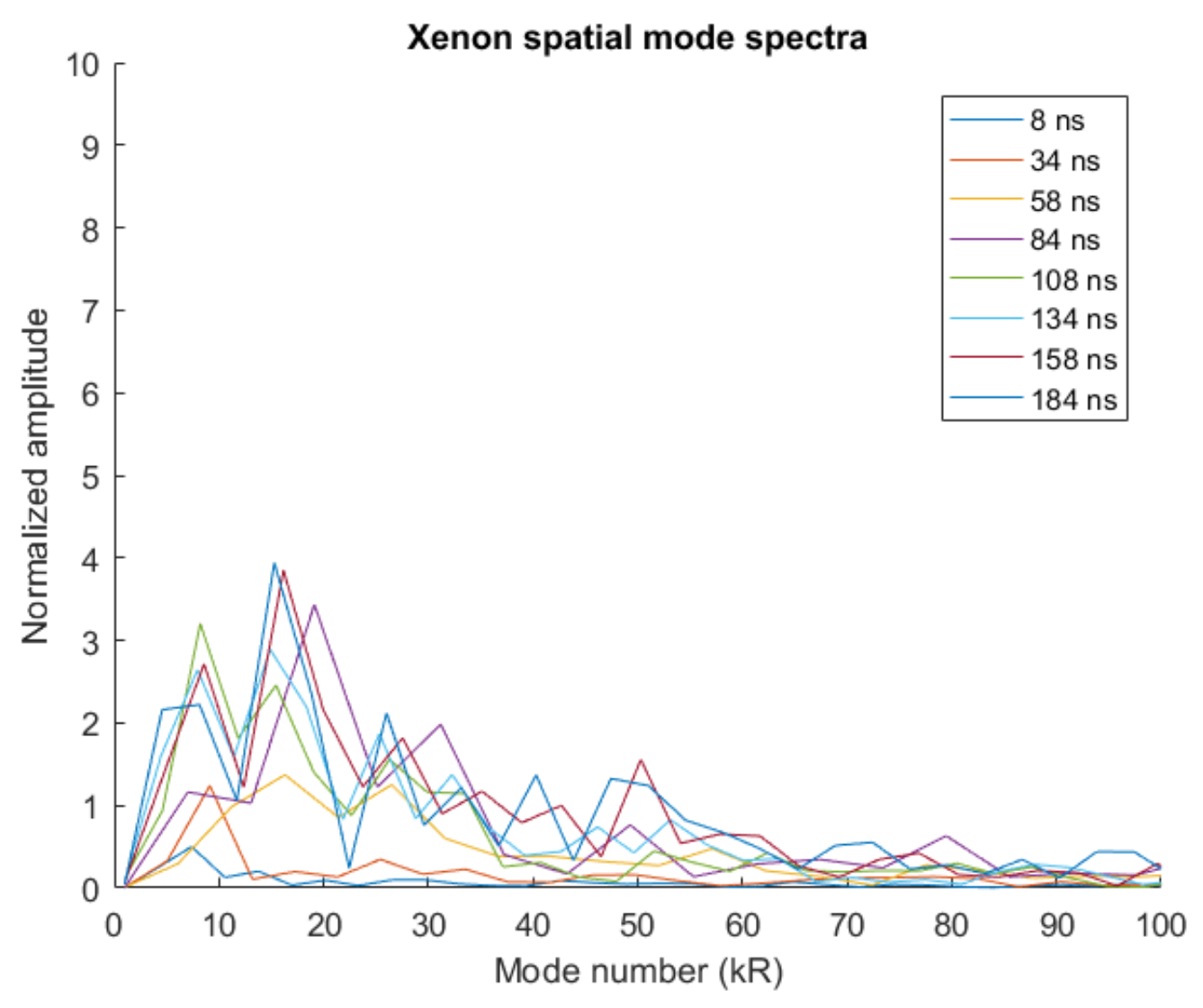

Figure 8. Spatial mode spectra of Xenon blast wave.

A plot of the time evolution of the dominant spectral mode is shown in figure 10 . Two features are salient. It is immediately apparent that the dominant spatial mode experiences a period of rapid growth from $\sim 50 \mathrm{~ns}$, and saturates roughly $50 \mathrm{~ns}$ thereafter. The perturbation continues to expand along with the blast wave (the dimensionless amplitude is given as $\delta R / R$ ), but is effectively frozen in after $\sim 100 \mathrm{~ns}$. We calculated the mode growth rate with the few points available and found that it follows a power law. This in agreement with Vishniac overstability predictions for perturbation growth. 


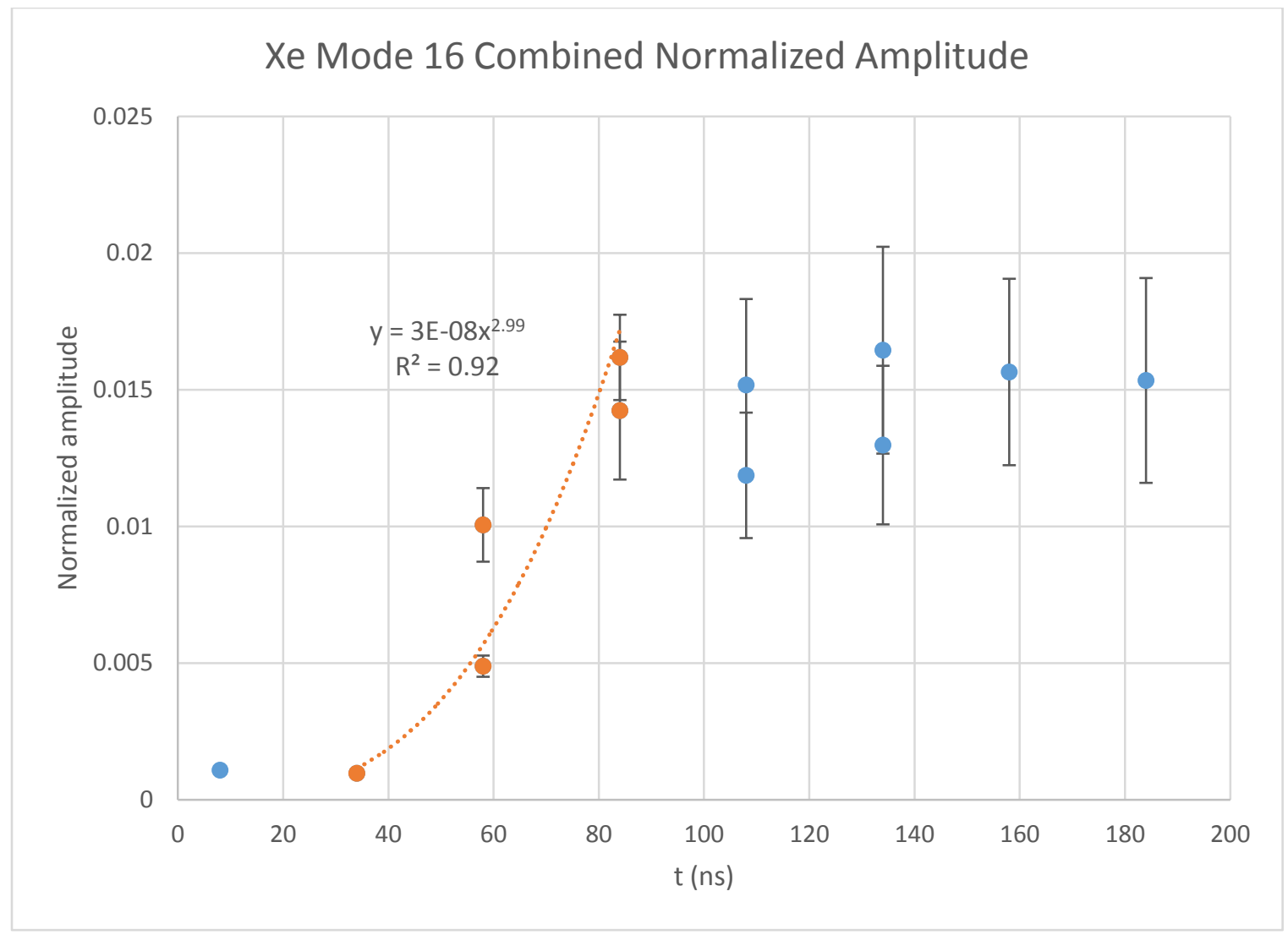

Figure 9. Time evolution of dominant spatial mode of xenon blast wave.

\section{Discussion}

As the first phase of the ZBL campaign was largely directed at the development and qualification of new diagnostic systems and target designs, it is useful to compare our results to the theory and to existing experimental work. The most useful theoretical description in the present context is the 1987 paper by Ryu and Vishniac [26]. This paper extends the thin-shell analysis derived above to realistic values of $\gamma$, and affords us a basis of comparison for our observations of the dominant mode number and growth rate. For an effective adiabatic index of 1.1, Ryu and Vishniac predicted a dominant mode number of about 30, with a power-law growth exponent of $\sim 0.5$. The corresponding figures from the experimental results of Grun et al. [2] show a dominant mode number of approximately 10-15 with a broad peak, and a growth exponent of $\sim 1.6$. Our results are much closer to those observed by Grun et al., although our measured growth exponent is still significantly higher.

In making the above comparison, a couple of caveats are in order. The theoretical mode spectrum is based on an effective $\gamma$ of 1.1, whereas our effective $\gamma$ is unknown with precision, but likely lower and time-dependent $[14,15]$. The theoretical numbers should therefore be regarded as an approximation, particularly in view of the fact that the mode spectrum is still calculated from a purely linear perturbation analysis. Additionally, although our experimental configuration is in many respects similar to that of Grun et al., we used a significantly greater drive energy at the same spatial scale. In principle, the strong drive approximation to the Euler similarity should mean that the flow variables in each experiment are well-scaled to each other. Nevertheless, the presence of perturbations at the same 
spatial scale but very different energy scales may have unforeseen consequences in the nonlinear regime.

We can therefore conclude that the mode spectrum of the instability is broadly consistent between theory and both experiments, although both experiments show substantially greater growth rates than predicted ( $1.5-3$ for the laser experiments, vs. $\sim 0.5$ for the linear theory). A plausible explanation for the disparity is that the experiments are driving the instability into the nonlinear regime. We note that the saturation of the instability observed at late times is consistent with the nonlinear analysis of MacLow and Norman [27].

To conclude, preliminary observations suggest magnetic effects on the stability and global structure of laser-launched radiative blast waves in dynamically significant magnetic field regimes. We find that a strong transverse magnetic field may shift the Vishniac overstability to longer wavelengths. The effects observed correspond qualitatively to predictions from the literature [1,7]. In order to determine the quantitative effects of the magnetic field on the spatial mode spectrum of the evolving blast wave, we have developed an experimental platform on a pulsed power compatible beamline on the Z-Beamlet laser which can resolve and decompose the spatial structure of the blast wave with precision. We have benchmarked an improved multiframe diagnostic chain on a pulsed-power capable beamline, and our preliminary results are in reasonable agreement with comparable experimental work and theory [1,2]. We are continuing the experiment on Z-Beamlet incorporating a 5-10 Tesla pulsed magnetic field in order to refine and extend our preliminary results.

\section{Acknowledgements}

This work was performed at the Center for High Energy Density Science at the University of Texas at Austin under NNSA Cooperative Agreement DE-NA0002008, and at Sandia National Laboratories. Sandia National Laboratories is a multi-program laboratory managed and operated by Sandia Corporation, a wholly owned subsidiary of Lockheed Martin Corporation, for the U.S. Department of Energy's National Nuclear Security Administration under contract DE-AC04-94AL85000. The data presented here is approved for unclassified unlimited release under R\&A SAND2015-1517 T. We also gratefully acknowledge the support of the Jane and Mike Downer Endowed Presidential Fellowship in Memory of Glenn Bryant Focht, and of the Michiro Naito Endowed Fellowship in Physics. Special thanks to Dr. Hernan Quevedo for a number of very helpful comments.

\section{References}

[1] E. Vishniac, The dynamic and gravitational instabilities of spherical shocks, Astrophys. J. (1983). http://adsabs.harvard.edu/full/1983ApJ...274..152V (accessed October 18, 2012).

[2] J. Grun, J. Stamper, C. Manka, J. Resnick, Instability of Taylor-Sedov blast waves propagating through a uniform gas, Phys. Rev. .... (1991). http://link.aps.org/doi/10.1103/PhysRevLett.66.2738 (accessed October 18, 2012).

[3] M.J. Edwards, a. J. MacKinnon, J. Zweiback, K. Shigemori, D. Ryutov, a. M. Rubenchik, K. a. Keilty, E. Liang, B. a. Remington, T. Ditmire, Investigation of Ultrafast Laser-Driven Radiative Blast Waves, Phys. Rev. Lett. 87 (2001) 1-4. doi:10.1103/PhysRevLett.87.085004.

[4] A.D. Edens, T. Ditmire, R.G. Adams, P.K. Rambo, L. Ruggles, I.C. Smith, J.L. Porter, Measurement of the Decay Rate of Single-Frequency Perturbations on Blast Waves, Phys. Rev. Lett. 95 (2005) 
1-4. doi:10.1103/PhysRevLett.95.244503.

[5] D. Ryutov, R. Drake, J. Kane, Similarity criteria for the laboratory simulation of supernova hydrodynamics, Astrophys. .... 518 (1999). http://iopscience.iop.org/0004-637X/518/2/821 (accessed October 18, 2012).

[6] D. Ryutov, Criteria for scaled laboratory simulations of astrophysical MHD phenomena, Astrophys. J. .... 127 (2000) 465-468. http://iopscience.iop.org/0067-0049/127/2/465 (accessed October 18, 2012).

[7] J. Blondin, E. Wright, Transition to the radiative phase in supernova remnants, Astrophys. ... 500 (1998) 342-354. http://iopscience.iop.org/0004-637X/500/1/342 (accessed October 26, 2012).

[8] D.F. Cioffi, C.F. McKee, E. Bertschinger, Dynamics of radiative supernova remnants, Astrophys. J. 334 (1988) 252. doi:10.1086/166834.

[9] Y. Zel'dovich, Y. Raizer, Shock waves and radiation, Annu. Rev. Fluid Mech. (1969). http://www.annualreviews.org/doi/pdf/10.1146/annurev.fl.01.010169.002125 (accessed October 18, 2012).

[10] R.E. Marshak, Effect of Radiation on Shock Wave Behavior, Phys. Fluids. 1 (1958) 24. doi:10.1063/1.1724332.

[11] J.F. Hansen, M.J. Edwards, D.H. Froula, G. Gregori, A.D. Edens, T. Ditmire, Laboratory observation of secondary shock formation ahead of a strongly radiative blast wave, Phys. Plasmas. 13 (2006) 22105. doi:10.1063/1.2168157.

[12] R. Chevalier, J. Imamura, Linear Analysis of an Oscillatory Instability of Radiative Shock Waves, Astrophys. J. 261 (1982) 543-549.

[13] E. Bertschinger, On the Structure and Stability of Radiative Shock Waves, Astrophys. J. 304 (1986) 154-177.

[14] J. Laming, J. Grun, Dynamical Overstability of Radiative Blast Waves: The Atomic Physics of Shock Stability, Phys. Rev. Lett. 89 (2002) 89-92. doi:10.1103/PhysRevLett.89.125002.

[15] J.M. Laming, J. Grun, Improved models for the dynamical overstability of radiative blast waves, Phys. Plasmas. 10 (2003) 1614. doi:10.1063/1.1556603.

[16] S. Reynolds, B. Gaensler, F. Bocchino, Magnetic fields in supernova remnants and pulsar-wind nebulae, Space Sci. Rev. (2012). http://www.springerlink.com/index/E861401351524011.pdf (accessed October 18, 2012).

[17] G. Toth, B. Draine, Oscillatory instability of radiative shocks with transverse magnetic field-Linear analysis and nonlinear simulations, Astrophys. J. (1993). http://adsabs.harvard.edu/full/1993ApJ...413..176T (accessed December 10, 2014).

[18] F. Heitsch, A.D. Slyz, J.E.G. Devriendt, L.W. Hartmann, A. Burkert, Magnetized Nonlinear Thin-Shell Instability: Numerical Studies in Two Dimensions, Astrophys. J. 665 (2007) 445-456. doi:10.1086/519513.

[19] K.W. Struve, J.L. Porter, D.C. Rovang, Megagauss Field Generation for High- Energy-Density Plasma Science Experiments, (2008). 
[20] J. Argo, J. Kellogg, D. Headley, LDRD final report on confinement of cluster fusion plasmas with magnetic fields., (2011). http://www.osti.gov/bridge/product.biblio.jsp?osti_id=1030401 (accessed October 18, 2012).

[21] L. Claus, L. Fang, R. Kay, M. Kimmel, J. Long, G. Robertson, M. Sanchez, J. Stahoviak, D. Trotter, J.L. Porter, An overview of the Ultrafast X-ray Imager (UXI) program at Sandia Labs, 9591 (2015) 95910P. doi:10.1117/12.2188336.

[22] P.K. Rambo, L.C. Smith, J.L. Porter, M.J. Hurst, C.S. Speas, R.G. Adams, A.J. Garcia, E. Dawson, B.D. Thurston, C. Wakefield, J.W. Kellogg, M.J. Slattery, H.C. Ives, R.S. Broyles, J. a Caird, A.C.

Erlandson, J.E. Murray, W.C. Behrendt, N.D. Neilsen, J.M. Narduzzi, Z-Beamlet: a multikilojoule, terawatt-class laser system., Appl. Opt. 44 (2005) 2421-30.

http://www.ncbi.nlm.nih.gov/pubmed/15861852.

[23] M. Wisher, Pulsed magnetic field generation for experiments in high energy density plasmas, University of Texas at Austin, 2014. http://repositories.lib.utexas.edu/handle/2152/25985 (accessed October 20, 2014).

[24] G.S. Settles, Schlieren and shadowgraph techniques: visualizing phenomena in transparent media, Springer Science \& Business Media, 2012.

[25] A. Edens, T. Ditmire, J. Hansen, Studies of laser-driven radiative blast waves, Astrophys. Sp. .... (2005) 39-47. http://www.springerlink.com/index/U2273528035747K8.pdf (accessed October $18,2012)$.

[26] D. Ryu, E. Vishniac, The growth of linear perturbations of adiabatic shock waves, Astrophys. J. (1987). http://adsabs.harvard.edu/full/1987ApJ...313..820R (accessed October 18, 2012).

[27] M. Mac Low, M. Norman, Nonlinear growth of dynamical overstabilities in blast waves., ... Mar. Elba Island, Italy, June 1-6, .... (1993).

http://books.google.com/books?hl=en\&lr=\&id=4g9sT_xZp3gC\&oi=fnd\&pg=PA256\&dq=Nonlinear +Growth+of+Dynamical+Overstabilities+in+Blast+Waves\&ots=IulReSS9nH\&sig=Ut84b9Vanzd_xd HWi9ahfj9Qcgk (accessed October 9, 2014). 